\title{
Ok, so who wants to colonise my gut? - Overview of Probiotics in certain Gastro-intestinal Disorders
}

\author{
GL Muntingh \\ Department of Pharmacology, School of medicine, Faculty of Health Sciences, University of Pretoria
}

*Corresponding author, email: george.muntingh@up.ac.za

\begin{abstract}
Probiotics are being used and perhaps more often than not as a treatment for a variety of gastrointestinal (GI) disorders. With increasing frequency, it is also being advocated as a treatment for several medical conditions, such as allergic diseases (atopic dermatitis, possibly allergic rhinitis), bacterial vaginosis, urinary tract infections, and prevention of dental caries or respiratory infections. Disturbances of bacterial microflora of the Gl tract may play an important role in the pathophysiology of some Gl disorders. It appears that probiotics being used as a treatment modality may have originated more than a century ago. It may restore normal microflora bacterial colonies and effect the functioning of the Gl tract by a variety of mechanisms. Probiotics are not currently regulated and only few authoritative randomised controlled trials exist investigating their efficacy in different Gl disorders. The efficacy of probiotics, as either a single strain or a combination of probiotics, has been assessed in antibioticassociated diarrhoea, Clostridium difficile colitis, infectious diarrhoea, ulcerative colitis, Crohn's disease, and irritable bowel syndrome, among other disorders. Although probiotics appear to be generally safe in an outpatient setting, no studies exist addressing the issue of safety specifically. Because most clinical trials in this respect are not congruent in design, many questions regarding use of probiotics in Gl disorders remain to be answered, such as most optimal doses, duration of treatment, physiological and immunological effects and efficacy of specific probiotics in specific disease states.
\end{abstract}

Keywords: Probiotics, Gastrointestinal disease, Safety, Clinical trials, Clinical effectiveness

\section{Introduction}

Some literature gives the word a full Greek etymology but it appears to be a composite of the Latin preposition pro ("for")

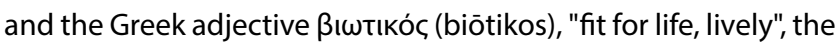
latter deriving from the noun $\beta$ íoc (bios, "life"). The term contrasts etymologically with the term antibiotic.

Recently, interest in human intestinal microbiota has supported numerous studies aimed at clarifying the role and dynamics of these micro-organisms. Today, we are aware that there are beneficial enteric micro-organisms and other potentially harmful ones and that the former can be used to influence or counteract the work of the latter. Some microorganisms, known as probiotics, when present in adequate amounts in the gut, are able to interact with the host influencing his/her immune response and produce a range of gastrointestinal and systemic benefits. Although most clinical studies on probiotics have been conducted only in the past few years, knowledge about probiotics dates back to the beginning of the past century when in 1908 the Nobel Prize laureate, Ilya llyich Mechnikov (Figure 1) attributed the longevity of Bulgarian peasants to their large consumption of fermented milk containing Lactobacillus bulgaricus and thereby introducing the concept (but not the term) of probiotics.

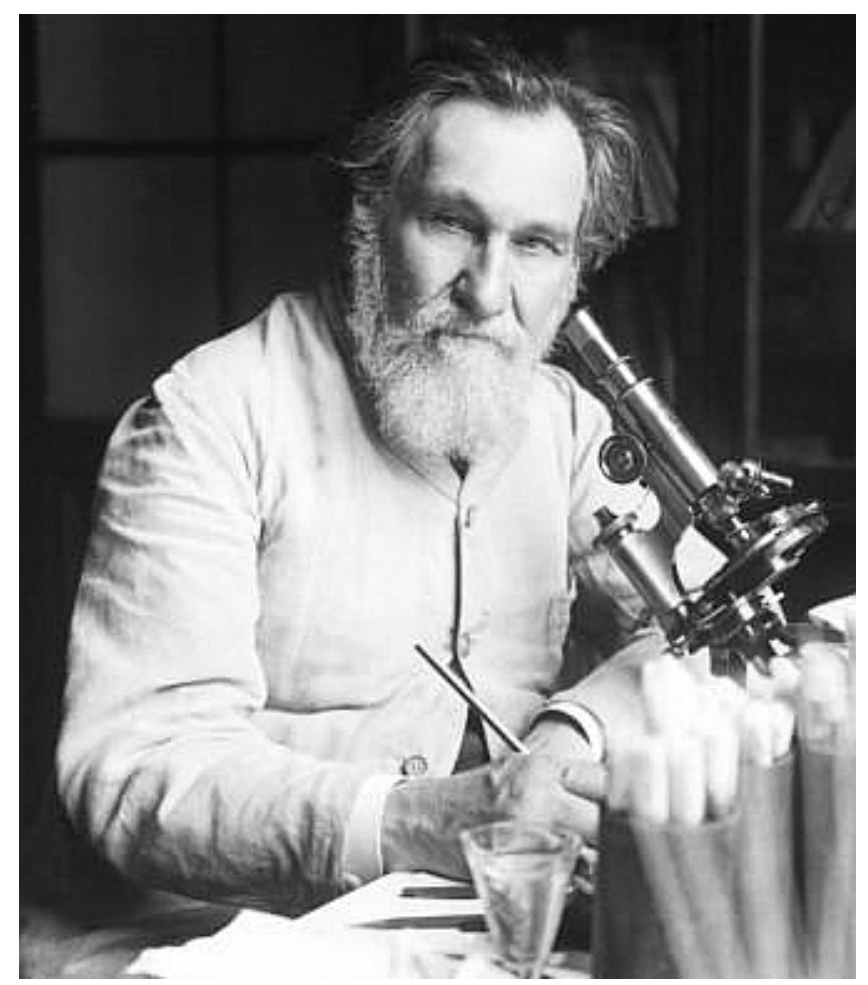

Figure 1. Ilya llyich Mechnikov

(https://commons.wikimedia.org/wiki/File:llya_llyich_Mechnikov_1913.jpg) 
In his observations, he states that the intestinal flora were affected by the intake of certain foods, which in turn stimulated the increase of useful bacteria within the intestine in order to replace the pathogenous species, which, on the contrary, caused damage. Over the years, many definitions have been used and formulated to describe probiotic bacteria. Today all these definitions are incomplete because they do not take into account the ability of these micro-organisms to generate an immune response within the host. Therefore, as proposed recently, the more comprehensive definition of probiotic bacteria agreed on is the following:

"Living micro-organisms, that when included in foods, can influence the composition and activity of the gut microbiota, modulate the inflammatory response, improve the non-specific intestinal barrier, and reinforce or modulate the mucosal and the systemic immune responses."

Many micro-organisms that compose the anaerobic intestinal microflora can be considered as potential probiotics, but only a few meet the advocated criteria. In particular, a probiotic strain should possess the following properties (Table 1):

\section{Table 1. Probiotic strain properties}

(1) High (bio)viability, therefore it must be able to reach the gut in a vital condition and this means that it must resist challenges posed by the gastrointestinal environment, e.g. low $\mathrm{pH}$, bile acids and pancreatic enzymes;

(2) Adhesion to the gut epithelium to cancel the flushing effects of peristalsis;

(3) Ability to persist in the intestine even if the probiotic strain cannot colonise the gut (continuous administration may be necessary);

(4) Be active and vital at the intestine environmental conditions;

(5) Be absolutely safe for human consumption;

(6) Be able to interact or to send signals to the immune cells by modulating the intestinal immune response;

(7) Be resistant to destruction by technological processes;

(8) Be stable during storage under normal conditions;

(9) Be able to influence the human metabolic activity. The ideal probiotics should be able to induce the best immune response without producing enteric changes in intestinal homeostasis.

Unfortunately, up until now, the mechanism of action of almost all probiotics has not yet been fully discerned and therefore understood.

A variety of probiotic micro-organisms have been studied both as single intended therapeutic agents and in combination. Currently, the more commonly used, in probiotic preparations, are listed in the Table 2.

A perusal of the literature suggests that the effects of probiotics' therapeutic or health claims can be summarised as listed in Table 3.
Table 2. Probiotic micro-organisms currently used in probiotic preparations

\begin{tabular}{lll}
\hline Lactobacillus sop. & \multicolumn{1}{c}{$\begin{array}{c}\text { Bifido } \\
\text { bacterium sop. }\end{array}$} & \multicolumn{1}{c}{ Other strains } \\
\hline acidophilus & longum & $\begin{array}{l}\text { Streptococcus } \\
\text { salivarius subsp. thermophilus }\end{array}$ \\
\hline acidophilus LA-1 & bifidum & Enterococcus faecium \\
\hline gasseri & breve & Propionilbacterium sop. \\
\hline crispatus & infantis & Saccharomyces boulardi \\
\hline amylovorus & animalis & Escherichia coli Nissle 1917 \\
\hline gallinarum & lactis & \\
\hline johnsonii & & \\
\hline casei sop. & & \\
\hline paracasei & & \\
\hline rhamnosus GG & & \\
\hline reuteri & & \\
\hline salivarius & & \\
\hline plantarum & & \\
\hline
\end{tabular}

Table 3. Probiotics' therapeutic or health claims

(1) Improvement of the normal gut microflora;

(2) Prevention of infectious diseases and food allergies;

(3) Reduction of serum cholesterol;

(4) Anticarcinogenic activity;

(5) Stabilisation of the gut mucosal barrier;

(6) Alleviation of chronic intestinal disease symptoms, and improved digestion of lactose in intolerant hosts:

(7) Assist in the synthesis of vitamins $\mathrm{K}_{\text {i }}$

(8) Prevention of Gl infections by virus, bacteria or fungi;

(9) Normalisation of intestinal motility;

(10) Immune adjuvant properties;

(11) Production of cytokines (cell signalling molecules);

(12) Participation in the prevention of osteoporosis;

(13) Dyspepsia, IBS and asthenia prevention.

\section{Mechanism of action}

There are various and possible mechanisms reported and purported to be involved in the therapeutic effects of probiotics. Figure 2 gives a schematic overview of these potential mechanisms whereby probiotic micro-organisms might influence the intestinal microbiota substrates. 




Figure 2: Schematic diagram illustrating potential or known mechanisms whereby probiotic bacteria might impact on the microbiota. These mechanisms include (1) competition for dietary ingredients as growth substrates, (2) bioconversion of, for example, sugars into fermentation products with inhibitory properties, (3) production of growth substrates, for example, EPS or vitamins, for other bacteria, (4) direct antagonism by bacteriocins, (5) competitive exclusion for binding sites, (6) improved barrier function, (7) reduction of inflammation, thus altering intestinal properties for colonization and persistence within, and (8) stimulation of innate immune response (by unknown mechanisms). IEC: epithelial cells, DC: dendritic cells, T:T-cells.

(Chapter from the book 'Probiotics and Prebiotics in Human Nutrition and Health':

http://www.intechopen.com/books/probiotics-and-prebiotics-inhuman-nutrition-and-health. With permission. Accessed 12 Sept 2017)

\section{Use of probiotics in gastrointestinal disorders}

\section{Acute gastrointestinal diseases}

\section{Infectious diarrhoea}

In infectious diarrhoea (also in its prevention), the use of probiotics may be appropriate in order to reduce the duration of symptoms. In this case, the most effective micro-organisms seem to be the Lactobacillus rhamnosus strain GG (LGG), Saccharomices boulardii and Lactobacillus paracasei (mainly in children). Also it appears that the bacteria are able to reduce the duration of infectious diarrhoea as well as its frequency.

Furthermore it appears that LGG, especially when concomitantly administered with an oral rehydration therapy (ORT), is able to produce major benefits in mild or moderate Rotavirus-diarrhoea. In this case, LGG also reduces the duration of viral elimination, restricting its spread.

However, it appears that the probiotics are not effective in non-Rotavirus diarrhoea. Although some studies suggest that Lactobacillus paracasei ST11 may have some efficacy in nonRotavirus diarrhoea, the definitive data are still lacking and insufficient in these particular forms of diarrhoea.

The reason it seems that LGG is effective in viral but lacks patency in bacterial diarrhoea is probably due to the ability of this pathogen to induce an over-expression of intestinal mucins (MUC-2, MUC-3) capable of performing a protective action during intestinal infections by polymerising into a gel which represents a real insoluble mucosal barrier able to protect the intestinal epithelium.

Unfortunately, it appears that the bacteria pathogen can circumvent this protective capacity through the production of a bacterial mucinase that is able to digest this insoluble protective layer.

\section{Antibiotic-associated diarrhoea (AAD)}

Diarrhoea caused by antibiotics represents a form that occurs in conjunction with oral antibiotics

use. The incidence and severity of AAD depend upon the specific type of antibiotic, the health of the host and exposure to pathogens. It results from an imbalance in the colonic microbiota caused by antibiotic therapy. The alteration in microbiota changes carbohydrate metabolism with decreased short-chain fatty acid absorption and an osmotic diarrhoea as a result. Another consequence of antibiotic therapy is overgrowth of potentially pathogenic intestinal organisms such as Clostridium difficile. It is believed, especially in the case of more severe episodes of diarrhoea, that $15-25 \%$ of cases of AAD are associated with the presence of Clostridium difficile (Clostridium difficile Associated Diarrhoea (CDAD))and for almost all cases of pseudomembranous colitis.

It appears that almost all oral antibiotics, particularly those with activity towards anaerobic bacteria, can cause AAD; investigations about the importance of a balance of intestinal flora have justified the use of probiotics in the treatment of this 
disease, and a number of studies have been performed on how to prevent AAD.

Meta-analyses of data have shown that some probiotic strains are associated with a reduction of AAD. In this case, the probiotic micro-organisms that have demonstrated some efficacy in the treatment of AAD were LGG, S. boulardii, B. lactis, and $S$. thermophilus. Supplementation significantly reduced diarrhoea, nausea, and taste disturbances associated with the treatment. Despite this, only S. boulardii has proved to be of clinical value in CDAD although other authors have shown that children with $A A D$ do greatly benefit from LGG supplementation.

However, in conflict, several other clinical studies have also documented a lack of efficacy of some probiotics (L. acidophilus, L. bulgaricus, and in a few cases, LGG and S. boulardii) in preventing AAD.

S. boulardii is a preparation of yeast that inhibits the growth of pathogenic bacteria, both in vivo and in vitro. Its optimum temperature is $37^{\circ} \mathrm{C}$, it resists digestion very well and, therefore exhibits good bioavailability and reaching the colon with preserved patency and vitality. S. boulardii does not seem to be influenced by antibiotic therapy. Once the therapy is completed, this organism is rapidly eliminated. S. boulardii significantly improved the fecal biostructure in patients with diarrhoea but had no influence on the faeces in healthy subjects.

To understand how $S$. boulardii can reduce recurrent episodes of $C D A D$, it should be taken into account that this yeast is able to inhibit $C$. difficile toxins $A$ and $B$ by issuing a protease that cleaves such toxins and their receptors at the brush border membrane.

\section{Chronic Inflammatory bowel diseases}

\section{Inflammatory bowel diseases (IBD)}

IBD are chronic diseases where genetically predisposed subjects with gut-associated lymphoid tissue (GALT) respond in an unusual way to the intestinal lumen antigens, including commensals. The main forms of IBD are Crohn's disease, Ulcerative Colitis, and Pouchitis.

Many investigations confirm that IBD are generally associated with a state of altered commensal gut microflora (commonly described as dysbiosis). It is known that intestinal dysbiosis are capable of triggering a pro-inflammatory effect even if they have not yet been identified as specific micro- organisms involved in the pathogenesis of this group of diseases.

The potential use of probiotics in IBD has been examined by several authors. Although experimental evidence, both in vitro and in vivo, support the use of probiotics in IBD, clinical trials have not provided definitive evidence regarding the use of probiotics in the treatment of IBD. The results seem to suggest that probiotics are most effective in IBD forms affecting the colon rather than the ileus. In particular, some studies suggest that probiotics are effective in prolonging the remission of Ulcerative Colitis and Pouchitis and appear to be less effective in the treatment of Crohn's disease and active Ulcerative Colitis.

\section{Crohn's disease (CD)}

Crohn's disease (CD) is considered as a predominantly T-lymphocyte driven disease characterised by the presence of a complex variety of interacting cytokines, chemokines, and other mediators produced by various other cell types.

The main theories of $C D$ pathogenesis suggest that the patients' T-cells, due to an unfortunate confluence of genetic and environmental factors, are inappropriately activated in the setting of an immune imbalance that leads to the classical chronic inflammation feature of this disorder.

There are various environmental factors, including diet, which may play an important role in the pathogenesis of $C D$. Among them, microbes seem to be a fundamental pathogenetic factor of $C D$.

In terms of the aetiology and pathogenesis of $C D$, three theories are proposed:

\section{An identified persistent pathogen;}

2. An abnormally permeable mucosal barrier leading to excessive bacterial translocation, and

3. An imbalance between the components of symbiotic and parasitic intestinal microbiota (dysbiosis).

Also considering that antibiotics are now part of the management of $C D$, especially the use of metronidazole and ciprofloxacin in perineal disease, probiotic therapy represents one possible approach to the prevention of relapse in CD patients.

Although several micro-organisms such as E. coli Nissle, Lactobacillus GG, and Saccharomyces boulardii have been assessed, there is currently no clear evidence whether these probiotic preparations are useful in active Crohn's disease treatment or in reducing the rate of

relapse. Moreover, no evidence could be sought that probiotics perform better than placebo or aminosalicylates in this setting.

Studies show the potential beneficial effect of synbiotics, but their role in anti-IBD therapy remains to be determined and therefore the use of probiotics as a maintenance therapy for medically or surgically induced remission in CD should be not recommended.

\section{Ulcerative colitis (UC)}

UC exhibits and includes a natural history of alternating periods of acute inflammatory disease and remissions.

The genetic predisposition to GALT dysregulation is the major hypothesis for UC aetiology. However, some epidemiological studies suggest that environmental factors can significantly alter the expression of these conditions.

In order to improve their quality of life, UC treatment traditionally, consisted of the use of aminosalicylates, to maintain the patients' remission as long as possible.

However, recent studies suggest that probiotic supplementation may represent a therapeutic alternative. 
A study of intestinal microflora on IL-10 elimination in mice revealed that as UC develops between the second and eighth week of life there is a decrease in the number of lactobacilli. It was shown, by repopulating the intestinal microflora of these mice with a supplementation of lactobacilli, that the histological severity of colitis was down-toned, and the number and adhesion on the mucosa and invasion by aerobic bacteria decreased considerably.

Several studies have evaluated the effects of synbiotic treatment in human UC. In particular, clinical trials on UC patients demonstrated the effectiveness of $E$. coli Nissle 1917 in maintaining remission in UC at a rate equivalent to treatment with mesalamine. In addition, other trials indicated beneficial effects in UC treatment with the use of: a combination of Bifidobacterium breve and B. bifidum; VSL\#3 B. longum, S. boulardii; a mixture of $B$. breve, B. bifidum and Lactobacillus acidophilus; LGG.

\section{Pouchitis}

Pouchitis is a common complication of a proctocolectomy with recanalisation with the ileum-anal pouch used in the treatment of UC and in essence considered a non-specific inflammatory disease of this ileal-pouch-anal-anastomosis (IPAA).

The causes of pouchitis have not been fully discerned thus far. However, findings of some studies are suggestive of a reduction in population density of lactobacilli and bifidobacteria in the pouch, indicating that these bacteria may play an important role in the development of this syndrome. Furthermore, the importance of bacteria is further emphasized by the evident efficacy of

antibiotics where most cases of pouchitis respond to treatment with antimicrobial agents.

The possible therapeutic role of probiotic therapy in pouchitis has been assessed in several clinical trials. The results overall have not demonstrated a clear superiority of some and between probiotic species although good evidence has emerged demonstrating the efficacy of the probiotic combination VSL\#3 (Lactobacillus plantarum, L. acidophilus, L. Delbrueckii subsp. bulgaricus, L. casei, Bifidobacterium longum, B. breve, B. infantis and Streptococcus salivarius subsp. thermophilus) and LGG in preventing primary pouchitis after IPAA surgery and in preventing relapse after antibiotic-induced remission.

\section{Other Gastro-intestinal conditions}

\section{Irritable bowel syndrome (IBS)}

Irritable bowel syndrome (IBS) is characterized by abdominal pain, flatulence, and irregular bowel movements and is the most frequently diagnosed gastrointestinal disease, and which affects about $12 \%$ of the European population.

While IBS is multifactorial, with no single aetiology to explain the disorder, many patients also experience comorbid behavioural disorders, such as anxiety or depression; thus, IBS is also described as a disorder of the gut-brain axis. Chronic stress can change motility and permeability throughout the gastrointestinal (Gl) tract. More recently, the role of the gut microbiota in the bidirectional communication along the gutbrain axis, and subsequent changes in behaviour, has emerged. Nevertheless, it is suspected that the increase in intestinal flatulence production that accompanies the syndrome is the result of an intestinal microflora alteration. More specifically, IBS has been associated with a greatly reduced colonic count of lactobacilli and bifidobatteri and an increase in anaerobic bacteria such as Clostridium sp. or other anaerobes (Bacteriodes sp. and Bifidobacterium sp.). On the other hand, studies, based on gene-sequencing of $16 \mathrm{~s}$ r-RNA sub-units of bacterial clones obtained by amplification with PCR of faecal genome showed that IBS patients have severely reduced sequences of Lactobacillussp. and Collinsellasp.

Studies with species of Lactobacillus have shown an improvement in pain and flatulence. In some studies, the supplement with Lactobacillus plantarum produced the reduction of intestinal pain and flatulence in IBS patients. However, to the contrary, two other trials with the same micro-organism did not show the same benefits.

Of interest, O'Mahoney et al. reported symptoms of IBS after exposureto Bifidobacterium infantis were alleviated, but not with Lactobacillus salivarus.

Satisfactory results have been obtained from supplementation with Saccharomyces boulardii, which, in two trials, was found to induce an overall improvement in symptoms of IBS patients.

Some work done by Zeng et al has shown that one of the important components in the mechanism of probiotics is the lactic acid of bacterial production. These authors have shown that treatment with bacterial lactic acid (produced by various micro-organisms such as Streptococcus thermophilus, Lactobacillus bulgaricus, Lactobacillus acidophilus and Bifid bacterium longum) induces the enhancement of mucosal barrier function in diarrhoeapredominant irritable bowel syndrome patients.

Although the studies are not easily comparable (and even at times discordant) due to differences in experimental protocol, doses, strains, and duration of therapy, in many cases the results suggest an improvement of symptoms, such as bloating, abdominal pain or alterations in colon transit. However, what seems clear is that supplementation with probiotics could be a promising therapy for patients suffering from IBS.

\section{Helicobacter pylori (HP)}

It is considered a risk factor of gastroduodenal ulceration, adenocarcinoma, and lymphoma.

In most subjects, the gastric HP colonisation is asymptomatic.

Currently, all symptomatic HP-positive patients (10-15\% infected population) are managed by simultaneous administration of antibiotics and a proton pump inhibitor. Despite this treatment, HP eradication is not guaranteed in all patients because of resistance or poor compliance, and children are prone to frequent relapses. Therefore, in recent years alternative approaches, including probiotic supplementation, have been 
taken into account to counteract the colonisation of HP in highrisk populations.

\section{Probiotics can counteract HP colonisation by:}

1. Inducing a decrease in the growth of bacteria pathogens;

2. Producing short-chain fatty acids which inhibit HP growth (especially acetic acid and lactic acid), in

a. dose-dependent manner;

\section{Producing bacteriocine;}

4. Stabilising the intestinal barrier function, and

5. Modulating mucosal inflammation due to prostaglandins increase.

Kim et al. have studied whether the addition of probiotics to proton pump inhibitor (PPI)-based triple therapy increases the likelihood of successful HP eradication. Their work showed that the administration of Lactobacillus acidophilus HY2177, Lactobacillus casei HY2743, Bifidobacterium longum HY8001, and Streptococcus thermophilus $B-1$ simultaneously in addition to a proton pump inhibitor (PPI)-based triple therapy increased the likelihood of HP eradication.

Of futher interest, Sabbi et al. showed that L. reuteri effectively suppresses HP becoming pathogenic in humans and decreases the occurrence of dyspeptic symptoms. Nevertheless, it does not seem to affect antibiotic therapy outcome.

In all cases, clinical trials in adults and children where HP was pathogenic suggest that probiotics do not eradicate HP, but can help to maintain low levels of this pathogen in the stomach.

\section{Summary and Further Directions}

The clinical evidence suggests there may be support for the use of probiotics as a valuable co-adjuvant therapeutic strategy for the prevention and treatment of gastro-intestinal diseases such as
IBD and IBS. Current evidence suggests this to be more significant in UC than in CD. However, more detailed mechanistic studies on the effectiveness of probiotics in these conditions are warranted to determine their potential beneficial effects. Therefore, more clinical trials with the use of appropriate molecular tools are necessary to determine which main outcomes and additional immune- and inflammation-associated variables are clearly influenced, and particularly the cause of these changes in the development of IBD.

This indicates the need for more randomised double-blind placebo-controlled trials with congruency in appropriate indication, doses and response, probiotic organisms and trial design.

Of importance are the interactions between the environment, the diet, antibiotics and the host and constitute one of the major issues in the development of IBD and IBS.

Major clinical trials should also study the mechanisms of action of probiotics using new molecular tools, such as the study of the microbiota changes using massive parallel sequencing (MPS), metabolomics, transcriptomics, and proteomics analyses of biopsies.

Following up with patients after the probiotic intervention to evaluate the persistence of beneficial effects needs to be done.

Ultimately, it is reasonable to assume that the "precautionary principle" should apply for probiotics use. Therefore, it is desirable that the use of probiotic microorganisms in the treatment of gastrointestinal disorders be limited only to those strains whose effectiveness and safety, in time and in ways that ensure the least possible impact on the intestinal microbiota, have been clearly demonstrated.

References available on request. 